\title{
SPALLATION NEUTRON SOURCE RING - STATUS, CHALLENGES, ISSUES, AND PERSPECTIVES*
}

\author{
Jie Wei ${ }^{\dagger}$ \\ for the Spallation Neutron Source Collaboration, USA
}

\begin{abstract}
The Spallation Neutron Source (SNS) ring is designed to accumulate beam pulses of $1.5 \times 10^{14}$ protons of $1 \mathrm{GeV}$ kinetic energy at a repetition rate of $60 \mathrm{~Hz}$ [1]. At such beam intensity and power, key design challenges include control of beam loss and radio-activation, construction of highquality large-aperture magnets and power supplies, design of robust injection and extraction systems, minimization of beam-coupling impedances, and mitigation of electroncloud effects. This paper discusses the status of the ring systems with emphasis on technical challenges and issues, and presents future perspectives towards a next-generation high-intensity facility.
\end{abstract}

\section{INTRODUCTION}

The SNS project is presently in the 5th year of a 7-year construction cycle [2]. The SNS ring, designed and constructed mainly by the Brookhaven National Laboratory, will accumulate pulses of $1.5 \times 10^{14}$ protons of $1 \mathrm{GeV}$ kinetic energy at a repetition rate of $60 \mathrm{~Hz}$ (Table 1) [1].

The primary concern is beam-loss induced radioactivation that can limit the ring's availability and maintainability. With a dedicated beam collimation in the ring at an efficiency above $90 \%$, the tolerable fractional beam loss is about $10^{-3}$ [3].

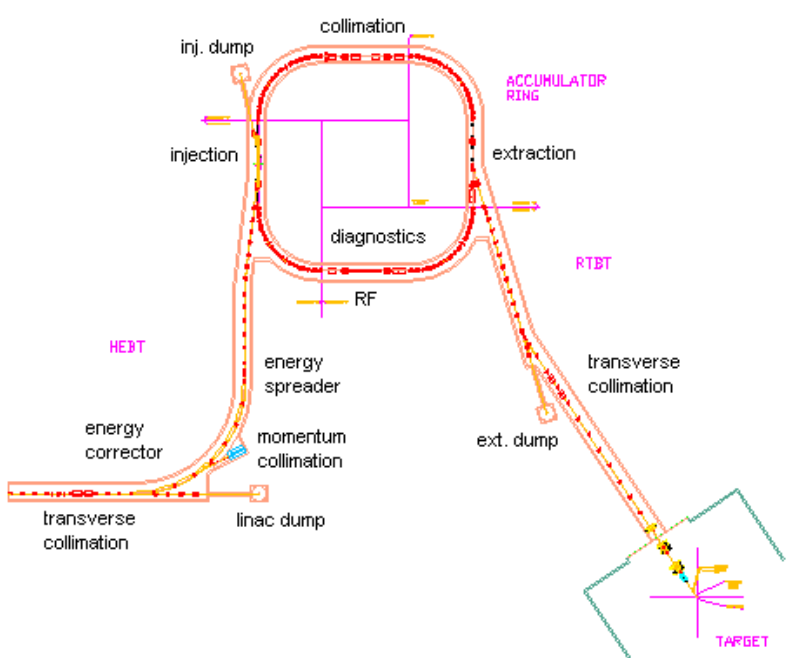

Figure 1: Layout of the SNS accumulator ring, the highenergy beam transport (HEBT), and the ring-to-target transport (RTBT).

* SNS is managed by UT-Battelle, LLC, under contract DE-AC0500OR22725 for the U.S. Department of Energy. SNS is a partnership of six national laboratories: Argonne, Brookhaven, Jefferson, Lawrence Berkeley, Los Alamos, and Oak Ridge.

†jwei@bnl.gov
Table 1: Major parameters of the SNS accumulator ring and its transport lines (HEBT and RTBT).

\begin{tabular}{ll}
\hline Quantity & Value \\
\hline Circumference & $248.0 \mathrm{~m}$ \\
HEBT, RTBT length & $169,151 \mathrm{~m}$ \\
Beam energy & $1 \mathrm{GeV}$ \\
Magnetic rigidity, $B \rho$ & $5.657 \mathrm{Tm}$ \\
Average beam power & $1.5 \mathrm{MW}$ \\
Repetition rate & $60 \mathrm{~Hz}$ \\
Number of protons per pulse & $1.6 \times 10^{14}$ \\
Revolution frequency & $1.058 \mathrm{MHz}$ \\
Peak RF voltage $(h=1,2)$ & $(40,20) \mathrm{kV}$ \\
No. of RF station (ring, HEBT, RTBT) & $4,2,0$ \\
Unnorm. emittance $\left(\epsilon_{x}+\epsilon_{y}\right.$, ,99\%) & $240 \pi \mu \mathrm{m}$ \\
Betatron acceptance & $480 \pi \mu \mathrm{m}$ \\
RF momentum acceptance & $\pm 1 \%$ \\
Transverse tunes ( $\left.\nu_{x}, \nu_{y}\right)$ & $6.23,6.20$ \\
Transition energy, $\gamma_{T}$ & 5.23 \\
Natural chromaticities $\left(\xi_{x}, \xi_{y}\right)$ & $-7.9,-6.9$ \\
No. of super-periods & 4 \\
No. of dipole (ring, HEBT, RTBT) & $39,9,1$ \\
Ring dipole field & $0.7406 \mathrm{~T}$ \\
Ring dipole gap height & $170 \mathrm{~mm}$ \\
No. of quad (ring, HEBT, RTBT) & $53,40,32$ \\
Ring quad inner diameter & $210-300 \mathrm{~mm}$ \\
No. of sextupole (ring, HEBT, RTBT) & $20,0,0$ \\
Sextupole inner diameter & $210-260 \mathrm{~mm}$ \\
No. of corrector (ring, HEBT, RTBT) & $61,18,17$ \\
No. of kicker (injection, extraction) & 8,14 \\
No. of scraper (ring, HEBT, RTBT) & $4,5,0$ \\
No. of absorber (ring, HEBT, RTBT) & $3,3,2$ \\
No. of vacuum pumps (ring, HEBT, RTBT) & $50,18,12$ \\
No. of power supply (ring, HEBT, RTBT) & $156,48,47$ \\
No. of BPM (ring, HEBT, RTBT) & $44,37,17$ \\
No. of loss monitor (ring, HEBT, RTBT) & $82,62,43$ \\
No. of current monitor (ring, HEBT, RTBT) & $2,5,5$ \\
No. of profile monitor (ring, HEBT, RTBT) & $4,13,8$ \\
Vacuum pressure, ring & $10^{-8} \mathrm{Torr}$ \\
\hline & \\
& \\
&
\end{tabular}

\section{ACCELERATOR DESIGN CHOICES}

\section{Accumulator ring}

During the first year of construction, a study was performed comparing the present structure of full-energy linac plus accumulator ring to a rapid-cycling-synchrotron (RCS) design: a $60 \mathrm{~Hz}, 400 \mathrm{MeV}$ linac feeds two, vertically stacked RCSs accelerating the proton beam to $2 \mathrm{GeV}$ energy. The biggest challenge to the RCS design is from the stringent $(1 \mathrm{~W} / \mathrm{m})$ beam-loss criterion: although relaxed by 
a factor of 5, still only $0.4 \%$ uncontrolled loss is allowed for a 2 MW beam power assuming 90\% collimation efficiency. On the other hand, among existing rings the lowest loss of about $0.3 \%$ is achieved at LANL's PSR, a $800 \mathrm{MeV}$ accumulator, as opposed to typical losses of a few to tens of percent in RCSs (e.g. ISIS, FNAL and AGS Boosters).

As opposed to the accumulator, the RCSs operating at 30 $\mathrm{Hz}$ require a high $\mathrm{RF}$ voltage (about $400 \mathrm{kV}$ per ring at 1.4 - $1.9 \mathrm{MHz}$ ) for fast acceleration, a large magnet aperture to accommodate the space charge at a lower energy, ceramic vacuum pipes with detailed RF shielding, and highperformance power supplies. Minimization of magnetic errors due to eddy current, ramping, saturation, and powersupply tracking is non-trivial. The study concluded that the required RCS design is technically more demanding and less cost effective [4].

Permanent magnets were considered as an option for the accumulator ring magnets. Electromagnetic magnets were chosen instead, given the uncertainty in the linac energy. This choice is especially appropriate to accommodate lateradopted superconducting-RF linac.

\section{Ring FODO-doublet lattice}

The four-fold symmetric ring lattice contains four dispersion-free straights, each housing injection, collimation, RF, and extraction (Fig. 1). Each achromatic arc consists of 4 FODO cells with $90^{\circ}$ horizontal phase advance.

After optimization, the ring lattice has doublet straights [1]. The lattice combines the FODO structure's simplicity and ease of correction with the doublet structure's flexibility for injection and collimation. Injection at a dispersionfree region allows independently adjustable painting in the transverse (with orbit bumps in the ring) and longitudinal (with an energy-spreading phase-modulated RF cavity in the HEBT) directions for a robust operation. The $12.5 \mathrm{~m}$ long uninterrupted straight section with a flexible phase advance further improves collimation efficiency. Comparing with the original all-FODO lattice, matching between the arcs and the straights increases the arc acceptance by $50 \%$ with the same magnet aperture (Fig. 2).

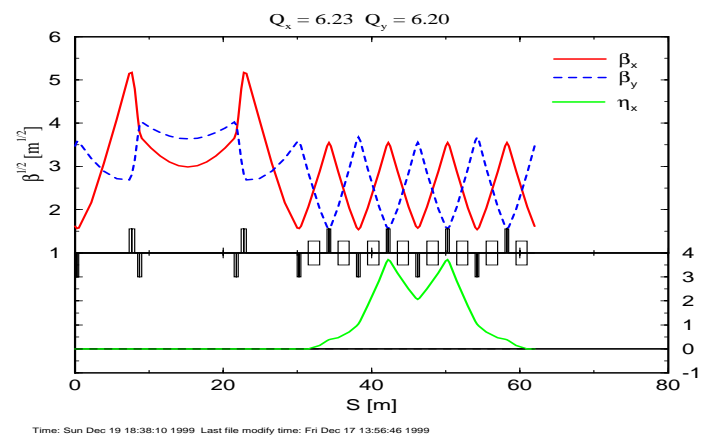

Figure 2: SNS ring FODO/doublet lattice.

\section{Lessons learned}

Solid-steel, as opposed to laminated-steel, was selected for most ring and transport magnet cores for cost savings.

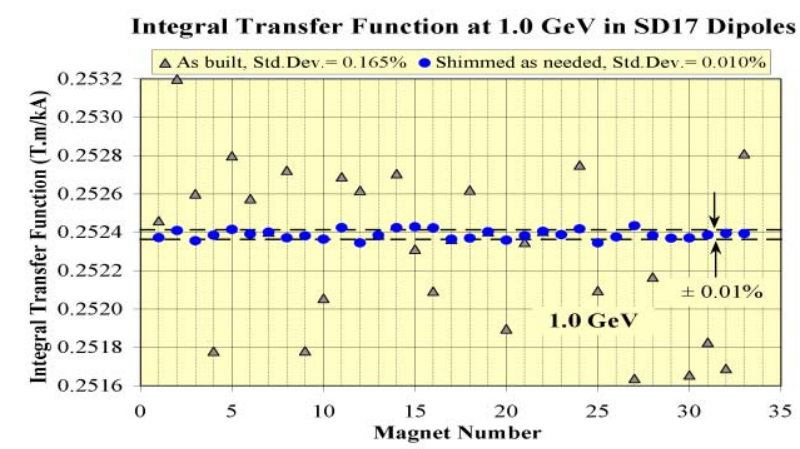

Figure 3: ITF Variation of the dipole magnets before and after shimming (courtesy P. Wanderer and A. Jain).

Individually, good field quality $\left(\sim 10^{-4}\right.$ relative error at full acceptance) is achieved. However, excessive (up to $0.25 \%$ ) magnet-to-magnet variation is found in the dipole integral transfer function (ITF) and its current dependence (Fig. 3) [5]. These dipoles are shimmed with iron sheets to achieve below $10^{-4}$ variation for $1 \mathrm{GeV}$ operation, and sorted according to $1.3 \mathrm{GeV}$ measurement data to minimize orbit corrector strength. Ring quadrupoles are partly sorted according to the power-supply family, and partly shimmed to achieve below $10^{-4}$ variation among each family.

The design does not allow in-situ baking of vacuum chambers that is needed for alternative NEG film coating.

\section{PHYSICAL CHALLENGES}

Main ring challenges include meeting the target requirements on the peak current density, minimizing uncontrolled beam loss, and controlling collective effects (space charge, instabilities, electron cloud [1].

\section{Beam loss}

Tables 2 and 3 list the expected controlled and uncontrolled beam loss. Beam collimation is performed at multiple locations (Fig. 1) to minimize the uncontrolled loss.

\section{Space charge}

The dominant collective effects are expected to be beam halo and beam loss generated by space-charge related res-

Table 2: Estimated controlled loss for a $2 \mathrm{MW}$ beam.

\begin{tabular}{lll}
\hline Mechanism & Location & Fraction \\
\hline HEBT: & linac dump & $10^{-5}$ \\
$\mathrm{H}^{0}$ from linac & H/V-collimator & $10^{-3}$ \\
linac transverse tail & L-collimator & $10^{-3}$ \\
linac energy jitter/spread & \\
Ring: & BIG kicker & $10^{-4}$ \\
beam-in-gap & collimator & $1.3 \times 10^{-5}$ \\
excited $\mathrm{H}^{0}$ at foil & injection dump & $10^{-2}$ \\
partial ionization at foil & injection dump & $10^{-2}$ \\
foil miss & collimator & $1.9 \times 10^{-3}$ \\
ring beam halo & collimator & $3 \times 10^{-6}$ \\
energy straggling at foil & & \\
RTBT: & & $10^{-5}$ \\
kicker misfiring & collimator & \\
\hline
\end{tabular}


Table 3: Estimated uncontrolled loss of a 2 MW beam.

\begin{tabular}{|c|c|c|c|}
\hline Mechanism & Location & Fraction & $\begin{array}{l}\text { Power } \\
{[\mathrm{W} / \mathrm{m}]}\end{array}$ \\
\hline \multicolumn{4}{|l|}{ HEBT: } \\
\hline $\mathrm{H}^{-}$magnetic strip. & all HEBT & $1.7 \times 10^{-6}$ & 0.02 \\
\hline $\begin{array}{l}\text { collimator out-scatt. } \\
\text { Ring: }\end{array}$ & achromat & $7.5 \times 10^{-6}$ & 0.1 \\
\hline $\mathrm{H}^{-}$magnetic strip. & inj. dipole & $1.3 \times 10^{-7}$ & 0.3 \\
\hline nucl. scatt. at foil & foil & $3.7 \times 10^{-5}$ & 2.5 \\
\hline collimation ineff. & all ring & $10^{-4}$ & 0.9 \\
\hline $\begin{array}{l}\text { RTBT: } \\
\text { nucl. scatt. at window }\end{array}$ & target window & $4 \times 10^{-2}$ & \\
\hline
\end{tabular}

onance crossing [6]. Other intensity-limiting mechanisms include electron-cloud effects and instabilities due to the extraction-kicker coupling impedance (Table 4).

The maximum incoherent space-charge tune spread is chosen to be 0.15 to avoid resonance-induced beam loss (Fig. 4). Transversely, the beam is painted to a full, unnormalized total emittance of $240 \pi \mu \mathrm{m}$. Longitudinally, a dual-harmonic RF system is used to achieve a bunching factor $\sim 0.45$, and HEBT energy spreader is used to paint a full momentum spread of $\pm 0.7 \%$ without enhancing tails.

\section{Impedance and instabilities}

Efforts are made to reduce the coupling impedance in a frequency range from about $200 \mathrm{~Hz}$ to above $50 \mathrm{MHz}$. 14 extraction kickers residing inside the vacuum chamber are major sources of transverse impedance. The impedance associated with the high- $\mu$ ferrite was reduced by terminating the pulse-forming network (PFN) circuit to $25 \Omega$, and by maximizing the width of the kicker module (Fig. 5).

\section{Electron-cloud effects}

Electron cloud remains to be an unresolved issue. Computer simulation indicates a trailing-edge electron concentration of peak value near $15 \mathrm{nC} / \mathrm{m}$, much higher than the value simulated for the PSR ring [8].

Mitigation measures involve suppressing electron generation and enhancing Landau damping [9]. Magnetron (dc) sputtering is used to coat the surfaces with $0.1 \mu \mathrm{m}$ TiN to reduce electron multipacting (Fig. 6). Electrons

Table 4: Collective effects and estimated thresholds for a 2 MW beam.

\begin{tabular}{ll}
\hline Mechanism & Threshold \\
\hline Trans. space charge & $\Delta \nu_{s c} \approx-0.2$ \\
Long. space charge & $15 \mathrm{kV}$ induced RF voltage \\
Trans. microwave instab. & $Z_{\perp} \approx 60 \mathrm{k} \Omega / \mathrm{m}$ \\
Long. microwave instab. & $\left|Z_{\|} / n\right| \approx 100 \Omega$ \\
Resistive wall & \\
$\quad(\xi=0)$ & $Z_{\perp} \approx 1.3 \mathrm{k} \Omega / \mathrm{m}$ at $200 \mathrm{kHz}$ \\
$\quad(\xi=-3)$ & $Z_{\perp} \approx 100 \mathrm{k} \Omega / \mathrm{m}$ at $200 \mathrm{kHz}$ \\
Electron-cloud & above $2 \mathrm{nC} / \mathrm{m}$ \\
\hline
\end{tabular}

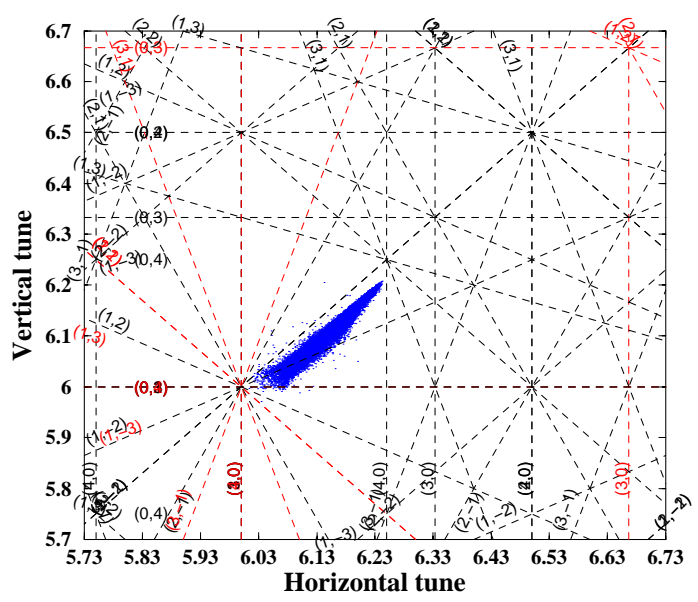

Figure 4: Transverse tune spread at the end of injection. Computer simulation is performed with UAL/ORBIT package [7]. Effects of space charge, transverse painting, chromaticity, kinematic non-linearity, fringe field, and magnetic manufacturing impections are included.
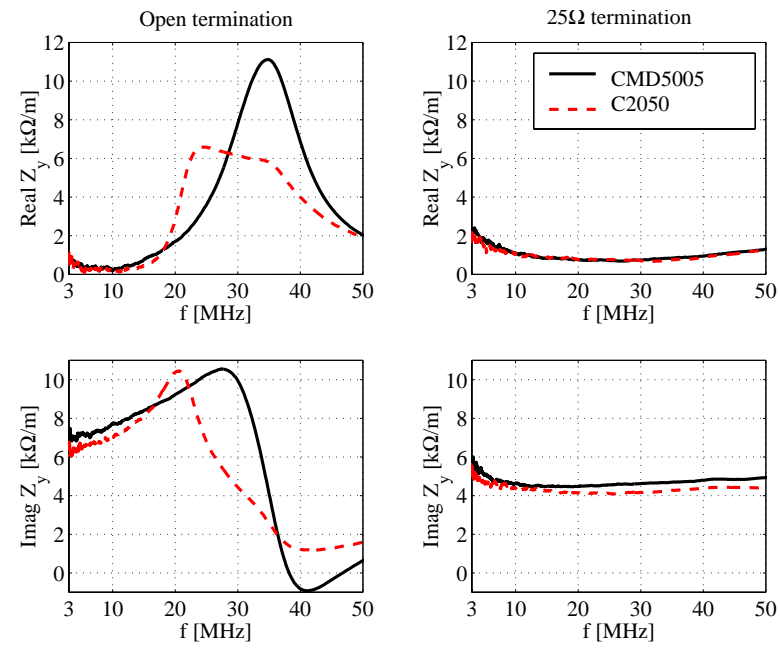

Figure 5: Comparison of measured coupling impedance for open and $25 \Omega$ PFN termination, and high (1600) and medium (100) permeability ferrite of the extraction kicker (courtesy D. Davino and H. Hahn).

in the injection region are guided to the collectors with a low backscattering yield. A beam-in-gap kicker ensures a clean beam-gap. Vacuum ports are screened, and steps in the vacuum pipe are tapered to reduce peaked electric fields causing electron emission. A good vacuum reduces electrons from gas ionization. Solenoids are wound in the collimation sections to reduce multipacting. Electrodes are installed in the injection region $(10 \mathrm{kV})$ and around the ring at BPM's $( \pm 1 \mathrm{kV})$ to clear the electron cloud. Electron detectors are installed at susceptible locations.

Enhancement of Landau damping includes a large vacuum chamber at locations of high dispersion and a large RF voltage to provide sufficient momentum acceptance, longitudinal painting to expand the momentum spread of the injecting beam, and lattice sextupole families for chromatic adjustments. Finally, a fast, wideband feedback system is 
planned to damp instabilities.

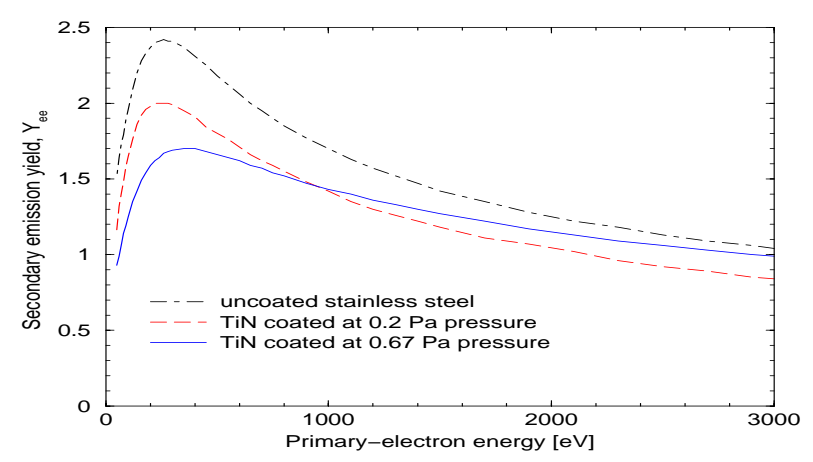

Figure 6: Measured secondary-electron yield for various coating conditions (courtesy P. He, H. Hseuh et al).

\section{TECHNICAL ISSUES \& STATUS}

\section{Injection}

For charge-exchange injection of an intense $\mathrm{H}^{-}$beam, free-hanging carbon and diamond foils are both developed to be mounted on a quick-exchange mechanism [10]. Stripped electrons are guided by the fringe field of tapered magnets and collected by heat-resistant C-C material attached to a water-cooled copper plate (Fig. 7). Both stripping foils and the electron catcher are monitored by video systems.

The transverse painting is achieved by 4 horizontal and 4 vertical dipoles with programmable power supplies. The measured minimum rise time (about $175 \mu \mathrm{s}$ ) is below the design value. The longitudinal painting is achieved by using a RF cavity located in the HEBT that is phase modulated with respect to the linac frequency.

\section{Collimation}

The $\mathrm{H}^{-}$beam in the HEBT is cleaned with stripping foils, and then a magnetic field separates the stripped beam from the original $\mathrm{H}^{-}$beam. The transverse collimation is located at the end of the linac upstream of the energy corrector, and the longitudinal collimation is located in the $90^{\circ}$ bend achromat.

The proton beam in the ring is cleaned transversely with a two-stage system, wherein the primary scraper scatters

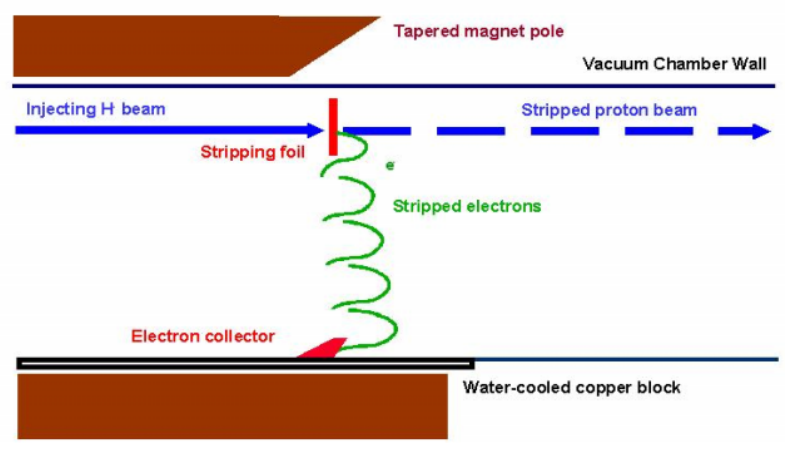

Figure 7: Injection area schematic layout. the beam and enhances its impact distance in the secondary collector, so achieving a cleaning efficiency above $90 \%$. The primary scraper consists of four tantalum blades, each $5 \mathrm{~mm}$ thick. They are spaced in 45 degree angles, adjustable to the varying needs of collimation aperture, and shielded for containing radioactivity. Due to reliability considerations, the secondary collectors are nonadjustable. Their vacuum chambers are made of doublelayered stainless-steel filled with helium gas between the layers to detect leaks [11]. Longitudinally, the proton halo moved into the beam gap is cleaned by the beam-in-gap kicker and collected by the two-stage collimation system during the last 100 turns before extraction.

\section{Extraction}

The beam is extracted during a single turn by being kicked vertically into the extraction channel of septum magnet. The septum bends the beam horizontally, and is also rotated to correct the vertical kicks. The kicker system consists of 14 individually powered modules, so that beam loss is negligible when one module fails. The PFNs are installed outside the ring tunnel for easy maintenance. The phase advance between the extraction kicker and the target is chosen so that the beam's position on target does not change if errors develop with the kickers.

To avoid complications due to rapid variations of the magnetic field, kicker magnets are made with a single-turn coil-winding and are placed inside the vacuum chamber. Good field quality is achieved with a high-permeability ferrite material. Saturable inductors isolate the influence of the PFN, shorten the rise time, and improve the flatness of the kicker-pulse's waveform. Beam density dependent closed-orbit deviation induced by the extraction kicker coupling-impedance at the given beam offset ("bananaclosed orbit") is about $1 \mathrm{~mm}$.

\section{Vacuum and chamber coating}

The design vacuum pressure is $(0.5 \sim 1) \times 10^{-7}$ Torr in HEBT to minimize $\mathrm{H}^{-}$stripping, $10^{-8}$ in the ring to minimize gas scattering and ion- and electron-induced desorptions, and $(0.5 \sim 1) \times 10^{-7}$ Torr in RTBT.

The entire inner surface of ring vacuum chamber and extraction kicker ferrite is coated with TiN [9]. Two layers of coating are applied to the ceramic chamber for injection kickers: a $1 \mu \mathrm{m}$-thick copper layer for by-passing the image charge, and a $0.1 \mu \mathrm{m}$-thick TiN layer for a low secondaryelectron yield, along with an exterior metal enclosure for $\mathrm{dc}$ current by-pass. This design allows the image-current passage above the lowest betatron sideband $(\sim 200 \mathrm{kHz})$ without degrading the magnetic-field penetration, eddy-current heating, and beam-induced heating.

\section{Magnet, correction, power supply}

Iterations are made on almost all kinds of magnets towards design expectation of magnetic accuracy at $10^{-4}$ for main dipoles and quadrupoles, $10^{-3}$ for sextupoles, chicane and transport magnets, and $10^{-2}$ for correctors. Some exceptions include a large $\left(0.19 \times 10^{-2}\right)$ systematic 20 th 
Table 5: Measured integral multipoles of the ring dipole magnet at a reference radius of $80 \mathrm{~mm}$. The systematic $b_{1}$ is due to tapered dipole ends.

\begin{tabular}{|l|ll|ll|}
\hline$n$ & \multicolumn{2}{|c|}{$b_{n}$} & \multicolumn{2}{c|}{$a_{n}$} \\
\hline & mean & S.D. & mean & S.D. \\
\hline 0 & 10000 & 0.0 & 0.0 & 0.0 \\
1 & -105.041 & 0.275 & -0.262 & 0.951 \\
2 & 0.163 & 0.433 & -0.020 & 0.494 \\
3 & 2.051 & 0.149 & -0.050 & 0.316 \\
4 & 1.143 & 0.200 & 0.000 & 0.113 \\
5 & 0.064 & 0.095 & 0.001 & 0.158 \\
6 & -0.300 & 0.120 & -0.017 & 0.105 \\
8 & -0.077 & 0.107 & 0.005 & 0.056 \\
10 & -0.176 & 0.129 & 0.003 & 0.028 \\
12 & 0.094 & 0.143 & -0.006 & 0.047 \\
\hline
\end{tabular}

Table 6: Measured multipoles of the ring quadrupole magnet at a reference radius of $80 \mathrm{~mm}$.

\begin{tabular}{|l|ll|ll|}
\hline$n$ & \multicolumn{2}{|c|}{$b_{n}$} & \multicolumn{2}{c|}{$a_{n}$} \\
\hline & mean & S.D. & mean & S.D. \\
\hline 1 & 10000 & 0.00 & - & - \\
2 & -0.30 & 1.11 & 0.41 & 1.20 \\
3 & -0.06 & 0.58 & 0.05 & 0.32 \\
4 & -0.01 & 0.20 & -0.04 & 0.23 \\
5 & 1.20 & 0.25 & -0.11 & 0.15 \\
9 & -0.73 & 0.02 & 0.00 & 0.06 \\
13 & -0.06 & 0.01 & -0.01 & 0.02 \\
\hline
\end{tabular}

pole on narrow-width quadrupoles used in the straights whose effects are negligible during the $1 \mathrm{~ms}$ accumulation, and large $(6 \%)$ decapole in multi-coil skew-sextupole correctors [12].

Four-family sextupoles are arranged in 4-fold ring lattice symmetry for chromaticity adjustment without compromising the dynamic aperture. The ring correction elements consist of horizontal and vertical dipoles, normal and skew quadrupoles, normal and skew sextupoles, and octupoles for orbit correction and decoupling, amplitude detuning, and resonance corrections. Octupoles can be rotated to act as skew-octupoles if needed. The reduction in quadrupole transfer function $(\sim-0.2 \%)$ caused by the interference from nearby sextupole/corrector is easily adjusted. Resonance correction strategy is developed in the presence of space charge [6].

There is 1 power supply for the main dipoles, 6 for the quadrupoles, 4 for the sextupoles, 115 for the correctors and chicane dipoles, 8 for the injection kickers, and 14 for the extraction kickers. Good rise/fall time and matching have been achieved on pulsed supplies (programmable injection and extraction) [13].

\section{RF system}

The HEBT has two RF cavities operating around linac frequency of $805 \mathrm{MHz}$, one for energy correction and the other for longitudinal painting. The ring dual-harmonic RF system maintains a gap for the rise time of the extraction kicker while maintaining low peak beam current and large momentum spread, reducing space charge stopband related losses and coherent instabilities [14].

\section{Diagnostics}

The ring and transport diagnostics consists of beam position monitor, loss monitor, current monitor, wire scanner, beam-in-gap monitor, foil/catcher video monitor, ionization profile monitor (IPM, Fig. 8), coherent/incoherent tune measurement system, and electron detector [15].

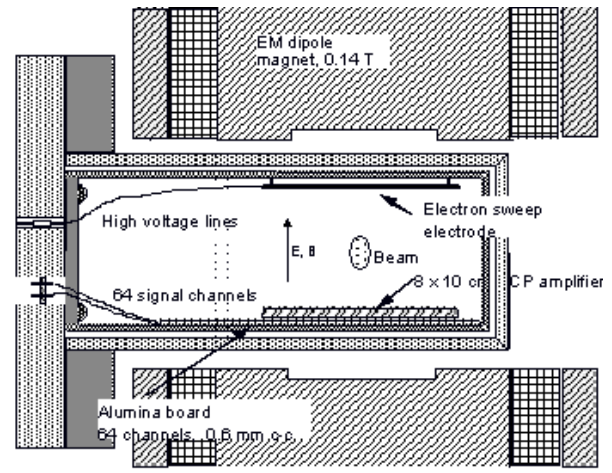

Figure 8: Ring IPM with sweeping electrodes, and with the multi-channel plates for electron collection recessed from the vacuum-chamber wall to avoid superfluous signals (courtesy R. Connolly).

\section{Reliability and availability}

Engineering design considers redundancy, radiation resistance, "active maintenance" capability (all-around cranes, quick-release flanges, quick-release water fittings, moveable shielding), and spares for areas of high radiation and activation.

\section{FUTURE PERSPECTIVES}

The ring and transport facility is designed with the potential to reach a beam energy up to $1.3 \mathrm{GeV}$ and a beam power beyond $2 \mathrm{MW}$, capable of supplying a second neutron target [1]. Space is reserved for two additional extraction kickers, and for the replacement of 2 injection-chicane dipoles to satisfy $\mathrm{H}^{0}$ stripping conditions.

We are indebted to the SNS teams and our collaborators for their devotion and contributions.

\section{REFERENCES}

[1] J. Wei et al, PRST-AB, 3 (1999) 080101; J. Wei, Rev. Mod. Phys. (Oct. 2003)

[2] N. Holtkamp, these proceedings

[3] N. Catalan-Lasheras et al PRST-AB 4 (2001) 010101

[4] J. Wei et al, EPAC00 (2000) 981

[5] P. Wanderer et al, these proceedings

[6] A. Fedotov, these proceedings

[7] N. Malitsky, these proceedings; J. Holmes, these proceedings

[8] M. Pivi, M. Furman, PRST-AB, 6 (2003) 034201

[9] M. Blaskiewicz; S.Y. Zhang et al; J. Wei et al, these proceedings; P. He et al, submitted to J. Vac. Sci. Tech. (2003)

[10] R. Shaw et al, these proceedings

[11] H. Ludewig et al; N. Simos et al, these proceedings

[12] J. Tuozzolo et al; N. Tsoupas et al; S. Badea et al; C. Pai, et al; J. Rank, et al, these proceedings

[13] J. Sandberg et al; W. Zhang et al, these proceedings

[14] A. Zaltsman et al; K. Smith et al, these proceedings.

[15] P. Cameron et al; M. Kesselman et al; D. Gassner et al; R. Connolly et al; R. Witkover et al, these proceedings. 